\title{
Effect of fuel type on solution combustion synthesis and photocatalytic activity of $\mathrm{NiFe}_{2} \mathrm{O}_{4}$ nanopowders
}

\author{
K. D. Martinson ${ }^{1}$, V.E. Belyak ${ }^{2}$, D. D. Sakhno ${ }^{2}$, \\ N. V. Kiryanov ${ }^{2}$, M. I. Chebanenko ${ }^{1}$, V. I. Popkov ${ }^{1}$ \\ ${ }^{1}$ Ioffe Institute, St. Petersburg, 194021, Russia \\ ${ }^{2}$ Saint Petersburg State Institute of Technology, 26 Moskovsky prospect, St. Petersburg, 190013, Russia \\ martinsonkirill@mail.ru
}

PACS 61.46.+w, 75.50.Bb, 75.60.-d

DOI 10.17586/2220-8054-2021-12-6-792-798

\begin{abstract}
Nickel ferrite nanopowders were obtained under conditions of solution combustion with the addition of various types of organic fuels (glycine, ascorbic acid, oxalic acid, and malic acid) and studied as photocatalysts in the photodegradation of methylene blue. Photocatalytic measurements were carried out under the action of visible light in model dye solutions. The highest photocatalytic activity was found in the case of a sample synthesized using malic acid as a fuel and in which the largest specific surface area $\left(128.1 \mathrm{~m}^{2} / \mathrm{g}\right)$ and the smallest particle size (18 nm) were observed. The obtained dependences of the photocatalytic activity of the synthesized samples demonstrate its strong dependence on morphological and structural parameters. Thus, with an increase in the particle size and a decrease in the specific surface area, a decrease in the efficiency of a photocatalyst based on nickel ferrite is observed. In addition, the use of various types of fuels made it possible to obtain aggregates of various shapes and sizes up to several tens of micrometers. The resulting rate constants were established to vary from 0.00756 to 0.00867 min ${ }^{-1}$.
\end{abstract}

Keywords: nickel ferrite, solution combustion synthesis, nanocrystals, photocatalysts, methylene blue.

Received: 6 September 2021

Revised: 1 December 2021

\section{Introduction}

In recent years, a large amount of research has been directed to the study of ferrites of various structures and compositions due to the combination of their unique magnetic and electromagnetic properties [1-3]. Thus, classical spinel ferrites are widely used in various fields of production of electrical equipment [4], magnetic storage media [5], and sensors [6]. In addition, to date, new areas of application of this type of materials have begun to appear, related to medicine, catalysis, biotechnology, etc. [7-9]. Special attention is paid to using ferrites as photocatalysts in various reactions of photodegradation under visible light $[10,11]$ due to the possibility of their magnetic separation caused by pronounced ferrimagnetic behavior [12]. The importance of these processes lies in the possibility of removing various types of organic pollutants from natural or industrial water sources and converting solar energy into chemical energy [13]. Most often, single-component semiconductors or conductors based on heterostructures such as zinc and titanium oxides together with bismuth oxide are used as materials for photocatalysis [14-16].

Among all the variety of spinel ferrites, nickel ferrite stands out, which has pronounced photocatalytic properties $[17,18]$. The functional properties of $\mathrm{NiFe}_{2} \mathrm{O}_{4}$ primarily depend on the features of its crystal structure. Nickel ferrite belongs to the class of inverted spinels and its general structure can be written as follows: $\left(\left[\mathrm{Fe}^{3+}\right]_{A}\left[\mathrm{Ni}^{2+}, \mathrm{Fe}^{3+}\right]_{B}\right)_{2} \mathrm{O}_{4}$. It is known that, depending on the synthesis conditions, $\mathrm{Ni}^{2+}$ cations can be located in both tetrahedral and octahedral positions, thereby changing the cation distribution [19]. This, in turn, can significantly affect both the magnetic and photocatalytic properties of the obtained samples [20]. To date, a large number of works have been published in which nickel ferrite nanoparticles were synthesized by sol-gel method [21], hydrothermal method [22], solution combustion method [23], mechanochemical synthesis [24], etc. A distinctive feature of the above methods is the ability to carry out the targeted synthesis of nanostructures with controlled particle size and shape as well as morphological and structural features [25]. Different varieties of solution combustion methods stand out, which, due to their simplicity [26] and the possibility of industrial scaling [27], are one of the most popular methods for obtaining nanostructured spinel ferrites of various compositions [28]. An important property of combustion methods is the large number of synthesis parameters, the variation of which makes it possible to change the structural and functional characteristics of the resulting powders across a wide range of values. In addition, this synthesis method is capable of providing the obtained products in high purity [29].

One of the key parameters of the solution combustion synthesis is the choice of organic fuel acting as both a chelating agent and a resource that ensures the course of the autoignition process. At present, a large number of various organic substances are known that can act as a fuel, among which glycine, ascorbic, oxalic, and malic acids are 
distinguished [30]. Each of these compounds has its effect on the initial reaction solution and provides different values of the crystallinity degree and particle size of the synthesized product. It is known that for catalysts and photocatalysts, morphological features are especially important, including the values of specific surface area and average particle size, on which their functional properties largely depend [31]. Often, within the framework of the classical single-phase method of solution combustion, even due to the selection of various types of organic fuels, it is difficult to obtain particles with a size less than $15-20 \mathrm{~nm}$ and a specific surface area of more than $40-50 \mathrm{~m}^{2} / \mathrm{g}$. To solve this problem, the authors of this work proposed a two-stage synthesis technique based on thermal treatment of X-ray amorphous combustion products, which consists in the synthesis of the initial powder with a significant lack of fuel and further mild heat treatment at 500-700 ${ }^{\circ} \mathrm{C}$. This technique has been repeatedly and successfully used to synthesize many systems of REE orthoferrites and spinel ferrites and has shown its high efficiency, including for the production of thermocatalytic and photocatalytic materials [32-34].

Therefore, the presented work is devoted to the study of the photocatalytic activity of pure nickel ferrites synthesized by the solution combustion method with the addition of various types of organic fuels (with their significant deficiency) and heat-treated at $600{ }^{\circ} \mathrm{C}$. All synthesized samples were investigated by XRD, SEM, EDX, and ASA methods, and their photocatalytic activity was tested using the model photodegradation of methylene blue.

\section{Experimental}

The following compounds were chosen as the initial reagents for the synthesis: $\mathrm{Ni}\left(\mathrm{NO}_{3}\right)_{2} \cdot 6 \mathrm{H}_{2} \mathrm{O}$ (puriss., NevaReactiv), $\mathrm{Fe}\left(\mathrm{NO}_{3}\right)_{3} \cdot 9 \mathrm{H}_{2} \mathrm{O}$ (puriss., NevaReactiv), $\mathrm{CH}_{2} \mathrm{NH}_{2} \mathrm{COOH}$ (puriss., NevaReactiv), $\mathrm{C}_{6} \mathrm{H}_{8} \mathrm{O}_{6}$ (puriss., NevaReactiv), $\mathrm{C}_{2} \mathrm{H}_{2} \mathrm{O}_{4}$ (puriss., NevaReactiv) and $\mathrm{C}_{4} \mathrm{H}_{6} \mathrm{O}_{5}$ (puriss., NevaReactiv). The starting reagents were taken following the reaction of the formation of the final product. Organic fuels were utilized in a 2.5-fold excess compared to reaction stoichiometry to obtain a completely X-ray amorphous powder.

The reaction solution was prepared by dissolving the reagents in $50 \mathrm{ml}$ of distilled water with constant mechanical stirring and heating to $25{ }^{\circ} \mathrm{C}$. To prevent the hydroxide precipitation of transition metals, the solution was acidified with a small amount of $3 \mathrm{M} \mathrm{HNO}_{3}$. After complete dissolution of the initial reagents, the solution obtained in this way was placed on a hot plate until the water was completely removed. After reaching the autoignition point, abundant evolution of gaseous reaction products and the formation of a brown solid product were observed. The synthesized powder was mechanically ground and thermally treated aerobically at $600{ }^{\circ} \mathrm{C}$ for 6 hours to remove unreacted organics and form a crystalline phase. The synthesis procedure is described in more detail in [35].

The obtained $\mathrm{NiFe}_{2} \mathrm{O}_{4}$ nanopowders were analyzed by powder X-ray diffractometry, scanning electron microscopy, energy-dispersive spectroscopy, and adsorption-structural analysis. Powder X-ray diffractometry was performed using a Rigaku SmartLab 3 diffractometer using the Rigaku SmartLab Studio II software package and an ICDD PDF 2 powder database. Scanning electron microscopy and energy dispersive spectroscopy were performed using a Tescan Vega 3 SBH instrument equipped with an Oxford INCX X-ray spectral microanalysis attachment. The adsorption-structural analysis was carried out using a Micromeritics ASAP 2020 instrument. Spectrophotometric measurements were carried out using a Shimadzu UV-1800 spectrophotometer. Methylene blue was chosen as a model dye. The $25 \mathrm{ppm}$ dye stock solution was prepared in $50 \mathrm{ml}$ of distilled water. Photocatalytic tests were carried out by adding a single number of synthesized powders to dye solutions and spectrophotometric analysis of a $5 \mathrm{ml}$ sample in 10-minute steps.

\section{Results and discussion}

Figure 1 shows diffraction patterns of nickel ferrite samples synthesized by the solution combustion method with the addition of various types of organic fuels (glycine, ascorbic acid, oxalic acid, and malic acid). The data obtained indicate that in all synthesized samples, a pure phase of nickel ferrite is observed. According to the Scherrer formula, the average crystallite size of the synthesized ferrites was calculated, which ranged from 18 to $27 \mathrm{~nm}$. The smallest size is observed in the case of the sample synthesized with the addition of malic acid $(18 \mathrm{~nm})$, while the largest is in the sample obtained using glycine $(27 \mathrm{~nm})$. It should be noted that the samples synthesized with the addition of oxalic acid and ascorbic acid had an average crystallite size close to that of malic acid, which is 20 and $21 \mathrm{~nm}$, respectively. The absence of visible displacements of the main diffraction maxima and the equality of the unit cell parameters of all synthesized powders (8.338(5)-8.339(1) ̊̊) make it possible to assert that their crystal structure is identical.

The elemental analysis and morphology of the synthesized $\mathrm{NiFe}_{2} \mathrm{O}_{4}$ samples were studied by energy dispersive analysis and scanning electron microscopy (SEM). SEM micrographs of the samples are shown in Fig. 2.

According to EDX data, all the obtained powders correspond to the calculated composition within the error of the determination method $(\mathrm{Fe}: \mathrm{Ni}=50: 50$ at. \%). According to the obtained micrographs, the morphology of nickel ferrite changes significantly depending on the type of fuel used. So, in the case of glycine, large aggregates with a size of 


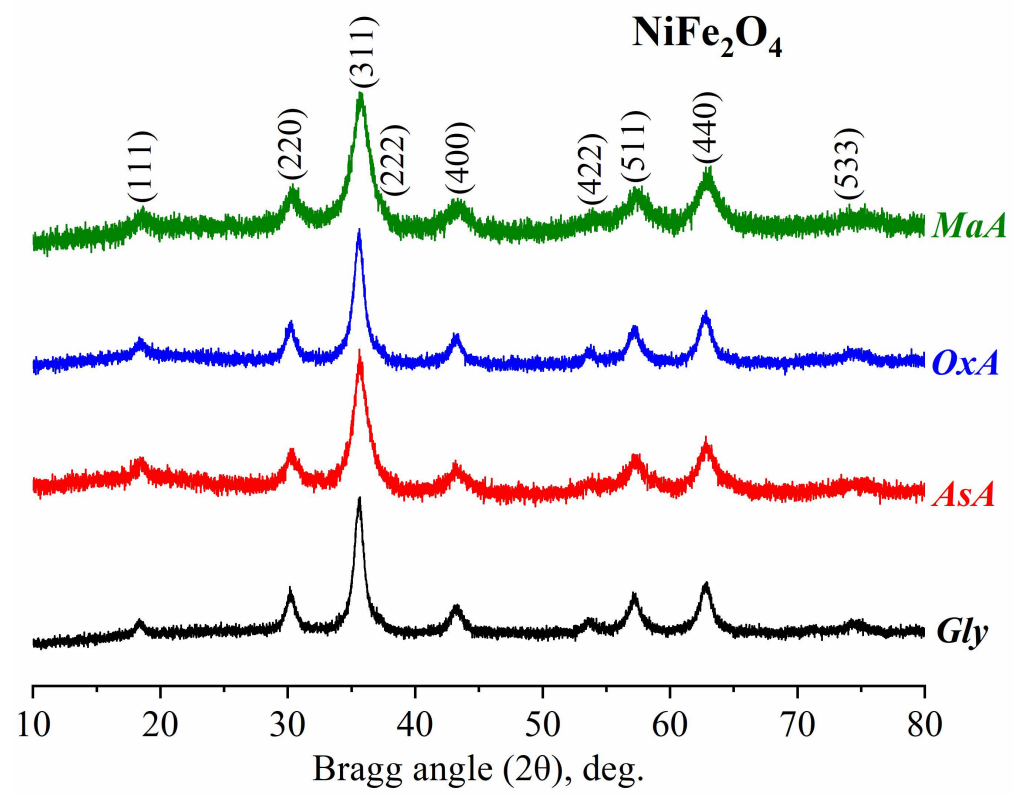

FIG. 1. PXRD patterns of the $\mathrm{NiFe}_{2} \mathrm{O}_{4}$ synthesized by the solution combustion method using glycine (Gly), ascorbic acid (AsA), oxalic acid (OxA), and malic acid (MaA)
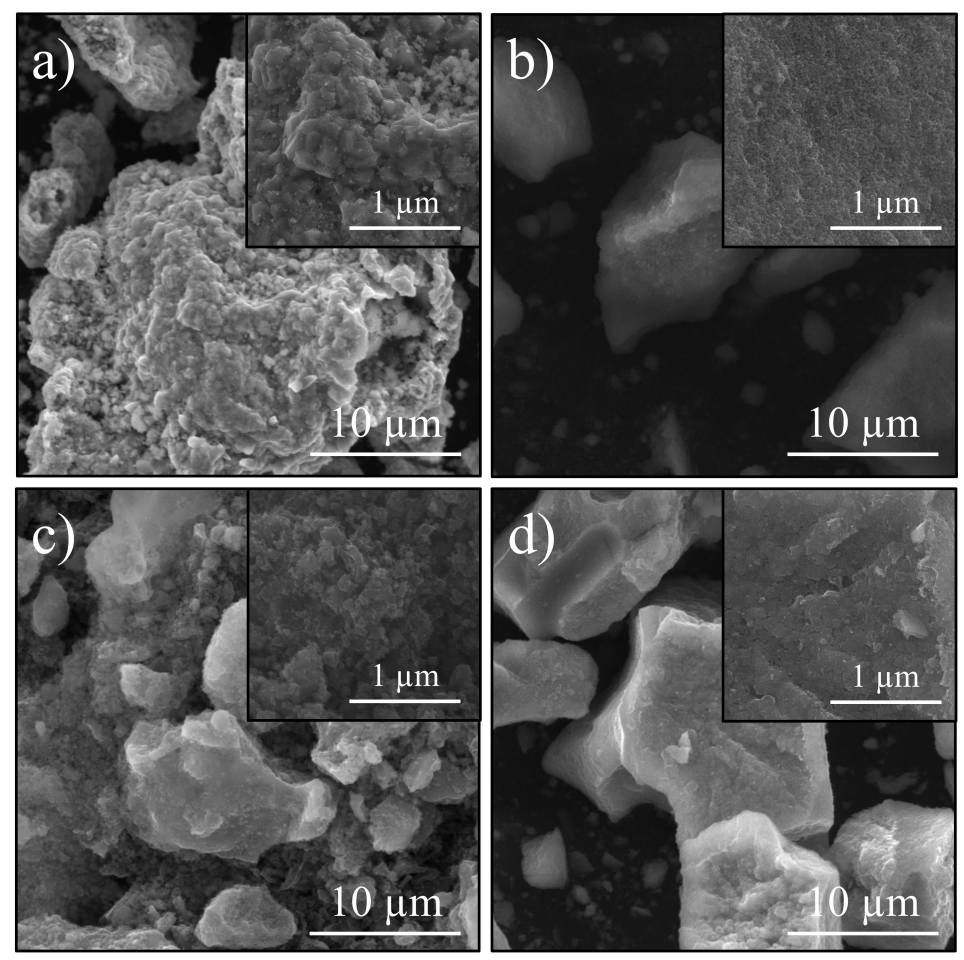

FIG. 2. SEM images of the $\mathrm{NiFe}_{2} \mathrm{O}_{4}$ nanopowders synthesized using solution combustion and different fuels - glycine (a), ascorbic acid (b), oxalic acid (c), and malic acid (d) 
10-20 microns are formed, consisting of well-sintered particles of submicron and nanometer sizes with a pronounced shape. In turn, the rest of the samples consist of particles of a much smaller size and have aggregates of a different shape and smaller size. An exception is a sample obtained with ascorbic acid, which has individual aggregates up to 35-40 microns in diameter, which, however, consist of particles of much smaller size than in the case of glycine.

The specific surface area of the synthesized powders was determined according to the data of low-temperature sorption-desorption of nitrogen, the results of which are shown in Fig. 3. It follows from the obtained data that all isotherms correspond to IV-type with hysteresis loops belonging to the H3-type according to the IUPAC classification. This appearance of the isotherms allows us to conclude that the obtained samples are highly porous, which is also confirmed by the data of scanning electron microscopy.

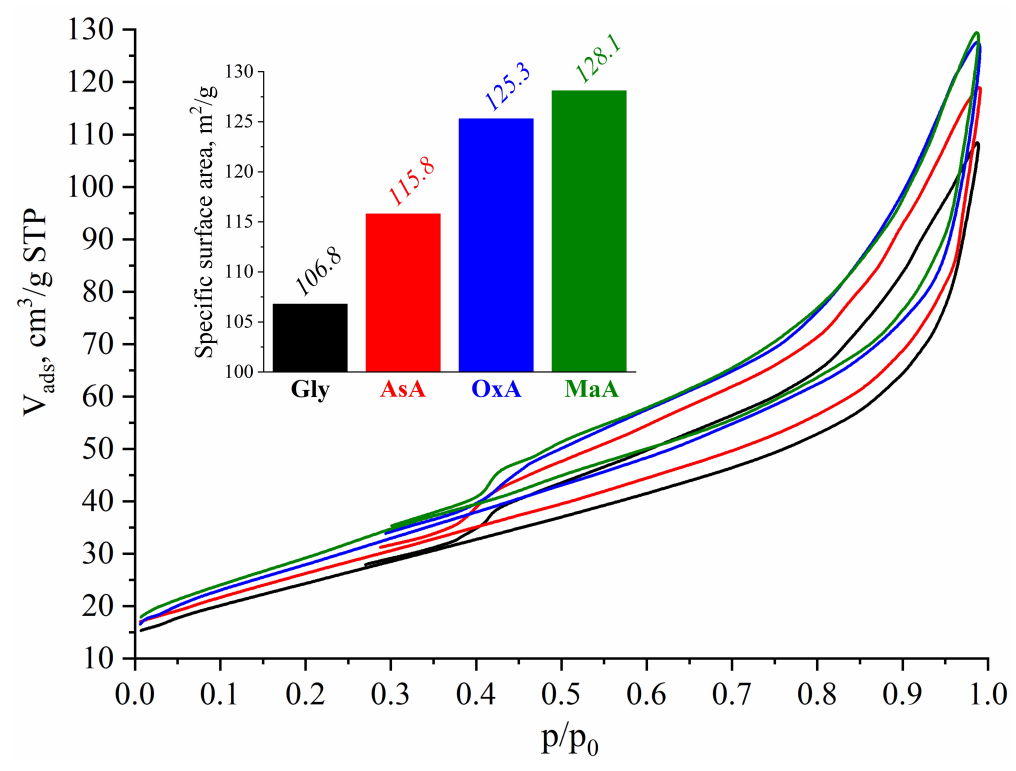

FIG. 3. Low-temperature nitrogen adsorption-desorption isotherms of synthesized nickel ferrite

The specific surface area calculated using the BET model was from 106.8 to $128.1 \mathrm{~m}^{2} / \mathrm{g}$, depending on the composition of the initial reaction medium. The smallest values were found in the case of nickel ferrite synthesized under the conditions of glycine-nitrate combustion, which agrees with the data of average crystallite size (the largest average crystallite size was observed for this sample). The most developed morphology and high specific surface area were observed for the samples synthesized with the addition of malic and oxalic acid $\left(128.1\right.$ and $125.3 \mathrm{~m}^{2} / \mathrm{g}$, respectively). It should be noted that this regularity is also explained by the average crystallite size, which reached the smallest values in these two cases. A slight decrease in the specific surface area for a sample burned in the presence of ascorbic acid is explained by the peculiarity of its morphology and the presence of large aggregates up to $40 \mu \mathrm{m}$ in size in its composition (Fig. 2b).

Photocatalytic reactions involving nickel ferrite are described in detail in the literature. Excited electrons present in the valence region pass into the conduction band, thus forming radicals of the $\mathrm{O}^{2-}$ type due to interaction with $\mathrm{O}_{2}$ molecules. At the same time, water molecules can react with the formed vacant holes in the valence band and form hydroxyl radicals of the $\cdot \mathrm{OH}$ type having high activity and capable of adsorbing various organic pollutants on their surface. In this work, methylene blue was chosen as a model dye to evaluate the photocatalytic activity of nickel ferrite nanopowders.

Figure 4 show the raw results of photocatalytic tests of the synthesized samples. According to the data obtained, one absorption peak of the dye at $660 \mathrm{~nm}$ was observed in all samples. With the inclusion of UV light, the peaks under the action of the present catalysts began to gradually decrease, which confirms the photocatalytic activity of all synthesized powders. The most intense decrease was observed in the initial period of UV treatment (transition from 0 to 10 minutes). Then, the decrease in the peaks slowed down, which is explained by the attainment of equilibrium and the peculiarity of the filling of active centers on the surface of the photocatalysts.

The percentage of decomposition of dyes ranged from 82.3 to $86.4 \%$, depending on the type of fossil fuel used to synthesize nickel ferrite nanoparticles (Fig. 5). The highest values were observed in samples obtained using malic acid and oxalic acid due to the high specific surface area and small particle size. In turn, the sample synthesized with glycine showed the worst indicators of photocatalytic activity, which, nevertheless, remained at a sufficiently high 


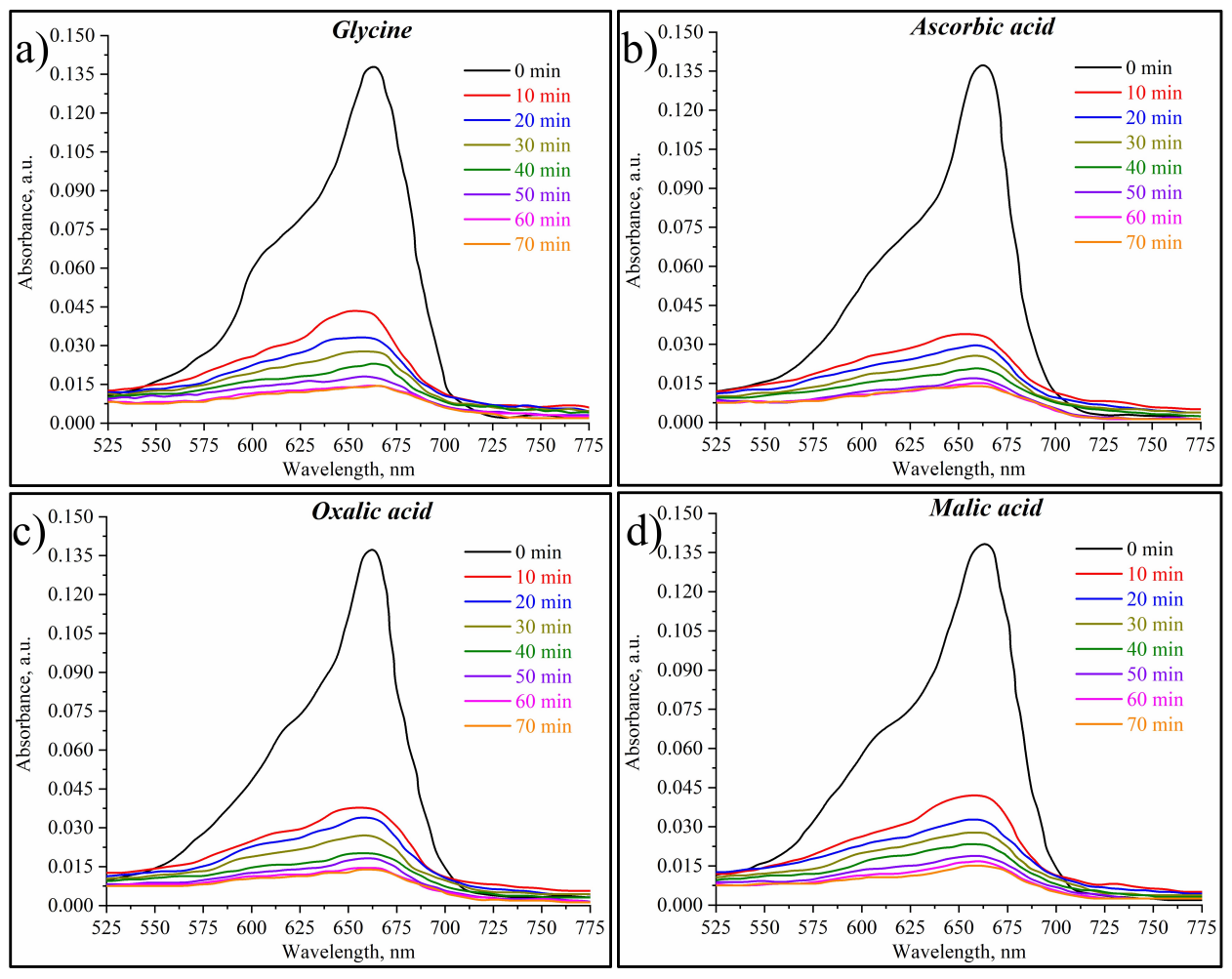

FIG. 4. Photodegradation of methylene blue over $\mathrm{NiFe}_{2} \mathrm{O}_{4}$ samples synthesized using: glycine (a), ascorbic acid (b), oxalic acid (c), and malic acid (d)
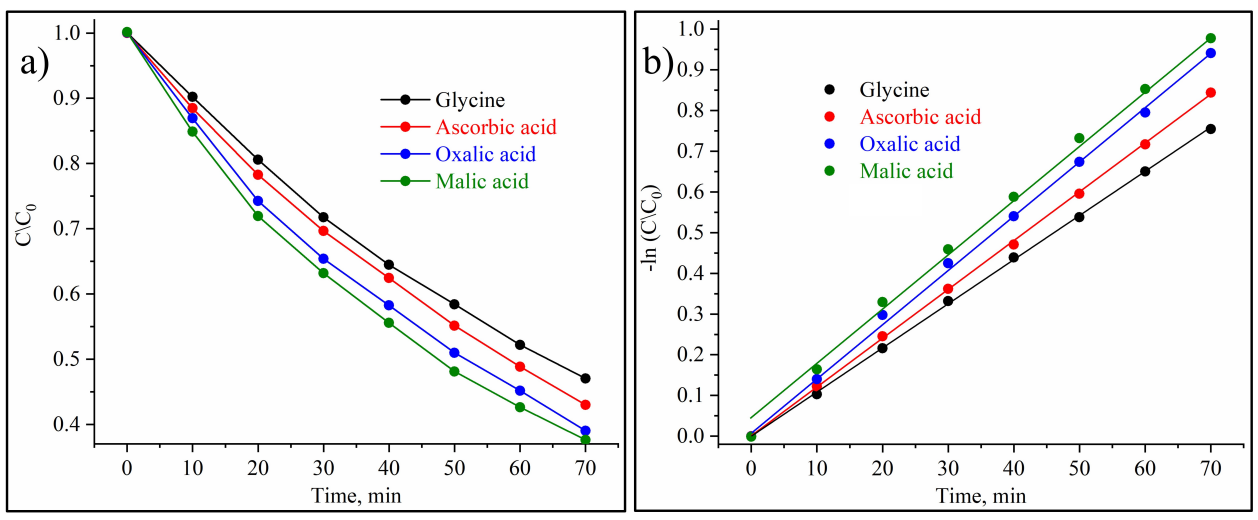

FIG. 5. Initial (a) and logarithmic (b) kinetic curves of methylene blue photodegradation over $\mathrm{NiFe}_{2} \mathrm{O}_{4}$ samples synthesized using different fuels

level. The band gap was determined by the diffusion reflectance spectroscopy and it turned out to be identical for all synthesized samples $(2.31 \mathrm{eV})$. The obtained values of the band gap are in good agreement with the literature [36].

The calculation of the pseudo-first-order rate constant (Fig. 6) showed that its highest values are achieved for the samples obtained under combustion conditions with the addition of malic acid $\left(0.00867 \mathrm{~min}^{-1}\right)$, while the smallest values are observed for the sample synthesized with glycine $\left(0.00756 \mathrm{~min}^{-1}\right)$. The obtained data are in good agreement with the data on the average crystallite size and specific surface area presented earlier and confirm that the samples synthesized with the addition of oxalic and malic acids are the most promising as photocatalysts.

\section{Conclusion}

Thus, in this work, nanostructured nickel ferrites were obtained using a solution combustion technique and various types of organic fuels (glycine, ascorbic acid, oxalic acid, malic acid). It was shown that the morphology significantly changes depending on the composition of the initial reaction medium and can correspond to aggregates in the range of 


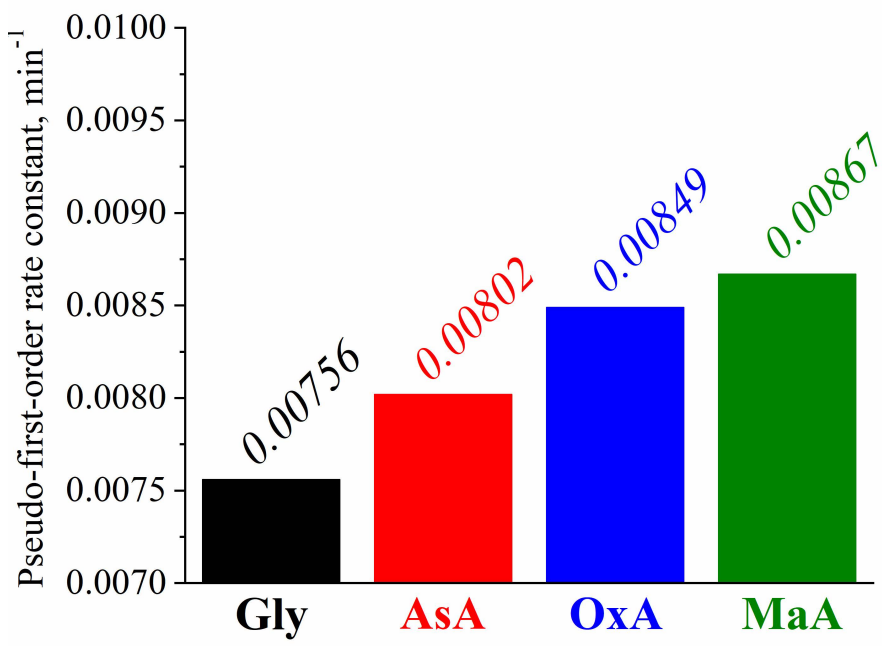

FIG. 6. Pseudo-first-order rate constant of nickel ferrite samples synthesized using glycine (Gly), ascorbic acid (AsA), oxalic acid (OxA), and malic acid (MaA)

values from 5 to $40 \mu \mathrm{m}$. The highest specific surface area and the smallest particle size were found in samples obtained using oxalic acid and malic acid. Analysis of photocatalytic activity showed high efficiency of all synthesized samples in photodegradation of methylene blue reactions.

\section{References}

[1] Kurian M., Thankachan S., Structural diversity and applications of spinel ferrite core-shell nanostructures - a review. Open Ceramics, 2021, 8, P. 100179.

[2] Ranga R., Kumar A, Kumari P, Singh P., Madaan V., Kumar K., Ferrite application as an electrochemical sensor: a review. Materials Characterization, 2021, 178, P. 111269.

[3] Houbi A., Aldashevich Z.A., Atassi Y., Telmanovna Z.B., Saule M., Kubanych K., Microwave absorbing properties of ferrites and their composites: a review. Journal of Magnetism and Magnetic Materials, 2021, 529, P. 167839.

[4] Almessiere M.A., Slimani Y., Trukhanov A.V., Sadaqat A., Korkmaz A.D., Algarou N.A., Aydin H., Baykal A., Toprak M.S., Review on functional bi-component nanocomposites based on hard/soft ferrites: structural, magnetic, electric and microwave absorption properties. Nano-Structures and Nano-Objects, 2021, 26, P. 100728.

[5] Harris V.G., Geiler A., Chen Y., Yoon S.D., Wu M., Yang A., Chen Z., He P., Parimi P.V., Zuo X., Patton C.E., Abe M., Acher O., Vittoria C., Recent advances in processing and applications of microwave ferrites. Journal of Magnetism and Magnetic Materials, 2009, 321, P. 20352047.

[6] Petrila I., Tudorache F., Effects of sintering temperature on the microstructure, electrical and magnetic characteristics of copper-zinc spinel ferrite with possibility use as humidity sensors. Sensors and Actuators A: Physical, 2021, 332, P. 113060.

[7] Rana G., Dhiman P., Kumar A., Vo D.-V. N., Sharma G., Sharma S., Naushad M., Recent advances on nickel nano-ferrite: a review on processing techniques, properties and diverse applications. Chemical Engineering Research and Design, 2021, 175, P. 182-208.

[8] Tikhanova S.M., Lebedev L.A., Martinson K.D., Chebanenko M.I., Buryanenko I.V., Semenov V.G., Nevedomskiy V.N., Popkov V.I., The synthesis of novel heterojunction h- $\mathrm{YbFeO}_{3} / \mathrm{o}-\mathrm{YbFeO}_{3}$ photocatalyst with enhanced Fenton-like activity under visible-light. New Journal of Chemistry, 2021, 45, P. 1541-1550.

[9] Amiri M., Eskandari K., Salavati-Niasari M., Magneticxally retrievable ferrite nanoparticles in the catalysis application. Advances in Colloid and Interface Science, 2019, 271, P. 101982.

[10] Kalam A., Al-Sehemi A.G., Assiri M., Gaohui D., Ahmad T., Ahmad I., Pannipara M., Modified solvothermal synthesis of cobalt ferrite $\left(\mathrm{CoFe}_{2} \mathrm{O}_{4}\right)$ magnetic nanoparticles photocatalysts for degradation of methylene blue with $\mathrm{H}_{2} \mathrm{O}_{2}$ /visible light. Results in Physics, 2018, 8 , P. 1046-1053.

[11] Irfan S., Zhuanghao Z., Li F., Chen Y.-X., Liang G.-X., Luo J.-T., Ping F., Critical review: bismuth ferrite as an emerging visible light active nanostructured photocatalyst. Journal of Materials Research and Technology, 2019, 8, P. 6375-6389.

[12] Shih Y.-J., Su C.-C., Chen C.-W., Dong C.-D., Synthesis of magnetically recoverable ferrite $\left(\mathrm{MFe}_{2} \mathrm{O}_{4}, \mathrm{M}=\mathrm{Co}, \mathrm{Ni}\right.$ and $\left.\mathrm{Fe}\right)$-supported $\mathrm{TiO}_{2}$ photocatalysts for decolorization of methylene blue. Catalysis Communications, 2015, 72, P. 127-132.

[13] Sonu, Sharma S., Dutta V., Raizada P., Hosseini-Bandegharaei A., Thakur V., Nguyen V.-H., VanLe Q., Singh P., An overview of heterojunctioned $\mathrm{ZnFe}_{2} \mathrm{O}_{4}$ photocatalyst for enhanced oxidative water purification. Journal of Environmental Chemical Engineering, 2021, 9, P. 105812.

[14] Sansenya T., Masri N., Chankhanittha T., Senasu T., Piriyanon J., Mukdasai S., Nanan S., Hydrothermal synthesis of ZnO photocatalyst for detoxification of anionic azo dyes and antibiotic. Journal of Physics and Chemistry of Solids, 2022, 160, P. 110353.

[15] Reddy K.M., Devaraju J., Kinetics of Photo Fenton Process and $\mathrm{Ag}_{-} \mathrm{TiO}_{2}$ photocatalyst under UV-light. Materials Today: Proceedings, 2019, 17, P. 234-238.

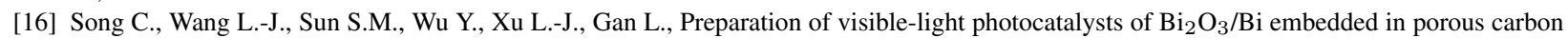
from Bi-based metal-organic frameworks for highly efficient Rhodamine B removal from water. New Carbon Materials, 2020, 35, P. 609-618. 
[17] Atacan K., Guy N., Cakar S., Ozacar M., Efficiency of glucose oxidase immobilized on tannin modified $\mathrm{NiFe}_{2} \mathrm{O}_{4}$ nanoparticles on decolorization of dye in the Fenton and photo-biocatalytic processes. Journal of Photochemistry and Photobiology A: Chemistry, 2019, 382, P. 111935.

[18] Shokri A., Using $\mathrm{NiFe}_{2} \mathrm{O}_{4}$ as a nano photocatalyst for degradation of polyvinyl alcohol in synthetic wastewater. Environmental Challenges, 2021, 5, P. 100332.

[19] Cvejic Z., Durdic E., Ivandekic G.I., Bajac B., Postolache P., Mitoseriu L., Srdic V.V., Rakic S., The effect of annealing on microstructure and cation distribution of $\mathrm{NiFe}_{2} \mathrm{O}_{4}$. Journal of Alloys and Compounds, 2015, 649, P. 1231-1238.

[20] Dumitrescu A.M., Samoila P.M., Nica V., Doroftei F., Iordan A.R., Palamaru M.N., Study of the chelating/fuel agents influence on NiFe ${ }_{2} \mathrm{O}_{4}$ samples with potential catalytic properties. Powder Technology, 2013, 243, P. 9-17.

[21] Majid F., Rauf J., Ata S., Bibi I., Malik A., Ibrahim S.M., Ali A., Iqbal M., Synthesis and characterization of $\mathrm{NiFe}_{2} \mathrm{O}_{4}$ ferrite: sol-gel and hydrothermal synthesis routes effect on magnetic, structural and dielectric characteristics. Materials Chemistry and Physics, 2021, 258, P. 123888

[22] Kesavan G., Nataraj N., Chen S.-M., Lin L.-H., Hydrothermal synthesis of $\mathrm{NiFe}_{2} \mathrm{O}_{4}$ nanoparticles as an efficient electrocatalyst for the electrochemical detection of bisphenol A. New Journal of Chemistry, 2020, 44, P. 7698-7707.

[23] Martinson K.D., Cherepkova I.A., Panteleev I.B., Popkov V.I., Single-step solution-combustion synthesis of magnetically soft NiFe $\mathrm{O}_{4}$ nanopowders with controllable parameters. International Journal of Self-Propagating High-Temperature Synthesis, 2019,28, P. $266-270$.

[24] Yang H., Zhang X., Ao W., Qiu G., Formation of $\mathrm{NiFe}_{2} \mathrm{O}_{4}$ nanoparticles by mechanochemical reaction. Materials Research Bulletin, 2004, 39, P. 833-837.

[25] Hajalilou A., Mazlan S.A., A review on preparation techniques for synthesis of nanocrystalline soft magnetic ferrites and investigation on the effects of microstructure features on magnetic properties. Applied Physics A, 2016, 122, P. 680.

[26] Martinson K.D., Cherepkova I.A., Sokolov V.V., Formation of cobalt ferrite nanoparticles via glycine-nitrate combustion and their magnetic properties. Glass Physics and Chemistry, 2018, 44, P. 21-25.

[27] Aruna S.T., Mukasyan A.S., Combustion synthesis and nanomaterials. Current Opinion in Solid State and Materials Science, 2008, 12, P. 44-50.

[28] Martinson K.D., Panteleev I.B., Shevchik A.P., Popkov V.I., Effect of the Red/Ox ratio on the structure and magnetic behavior of Li $\mathrm{i}_{0.5} \mathrm{Fe}_{2.5} \mathrm{O}_{4}$ nanocrystals synthesized by solution combustion approach. Letters on Materials, 2019, 9, P. 475-479.

[29] Khort A., Roslyakov S., Loginov P., Solution combustion synthesis of single-phase bimetallic nanomaterials. Nano-Structures \& NanoObjects, 2021, 26, P. 100727.

[30] Lazarova T., Georgieva M., Tzankov D., Voykova D., Aleksandrov L., Cherkezova-Zheleva Z., Kovacheva D., Influence of the type of fuel used for the solution combustion synthesis on the structure, morphology and magnetic properties of nanosized $\mathrm{NiFe}_{2} \mathrm{O}_{4}$. Journal of Alloys and Compounds, 2017, 700, P. 272-283.

[31] Kharisov B.I., Dias H.V.R., Kharissova O.V., Mini-review: ferrite nanoparticles in the catalysis. Arabian Journal of Chemistry, 2019, 12, P. 1234-1246.

[32] Popkov V.I., Martinson K.D., Kondrashkova I.S., Enikeeva M.O., Nevedomskiy V.N., Panchuk V.V., Semenov V.G., Volkov M.P., Pleshakov I.V., SCS-assisted production of $\mathrm{EuFeO}_{3}$ core-shell nanoparticles: formation process, structural features and magnetic behavior. Journal of Alloys and Compounds, 2021, 859, P. 157812.

[33] Martinson K.D., Ivanov A.A., Panteleev I.B., Popkov V.I., Effect of sintering temperature on the synthesis of LiZnMnFe microwave ceramics with controllable electro/magnetic properties. Ceramics International, 2021, 47, P. 30071-30081.

[34] Martinson K.D., Sakhno D.D., Belyak V.E., Kondrashkova I.S., Ni $\mathrm{N}_{4} \mathrm{Zn}_{0.6} \mathrm{Fe}_{2} \mathrm{O}_{4}$ nanopowders by solution-combustion synthesis: influence of Red/Ox ratio on their morphology, structure, and magnetic properties. International Journal of High-Temperature Synthesis, 2020, 29, P. 202-207.

[35] Martinson K.D., Kozyritskaya S.S., Panteleev I.B., Popkov V.I., Low coercivity microwave ceramics based on LiZnMn ferrite synthesized via glycine-nitrate combustion. Nanosystems: Physics, Chemistry, Mathematics, 2019, 10, P. 313-317.

[36] Amulya M.A.S., Nagaswarupa H.P., Kumar M.R.A., Ravikumar C.R., Prashantha S.C., Kusuma K.B., Sonochemical synthesis of NiFe ${ }_{2}$ O $_{4}$ nanoparticles: characterization and their photocatalytic and electrochemical applications. Applied Surface Science Advances, 2020, 1, P. 100023. 